\title{
Telemedicine and Stroke Care Services: Experience from Italian Hospitals
}

\author{
Mariangela Panebianco $^{1 *}$, Domenico Restivo ${ }^{2}$, Pavone Antonino ${ }^{2}$, Patrizia Perrone ${ }^{3}$, and Chiara \\ Zavanone $^{4}$ \\ ${ }^{1}$ Department of Molecular and Clinical Pharmacology, University of Liverpool, UK \\ ${ }^{2}$ Neurology Department, Garibaldi Hospital, Italy \\ ${ }^{3}$ Neuroscience Department, ASST Ovest Milanese, Italy \\ ${ }^{4}$ Neurology Department, Pierre et Marie Curie University, France
}

*Corresponding author: Mariangela Panebianco, Department of Molecular and Clinical Pharmacology, Institute of Translational Medicine, University of Liverpool, UK.

To Cite This Article: Mariangela Panebianco, Telemedicine and Stroke Care Services: Experience from Italian Hospitals. 2020 - 8(1). AJBSR. MS.ID.001224. DOI: 10.34297/AJBSR.2020.08.001224.

Received: 淎 February 28, 2020; Published: 眥 March 09, 2020

\begin{abstract}
Introduction: According to the American Telemedicine Association, "Telemedicine" is the exchange of medical information from one site to another using electronic communication, such as telephone, internet, or videoconference. The application of telemedicine for care of acute strokes, called "Telestroke", was a natural progression from general telemedicine, because of shortage of vascular neurologists and recent advances in technology. The use of telestroke across the United States and Canada occurred in the late 1990s and early 2000s, but in Italy it comes later. It is one of the most frequently used and rapidly expanding applications of telemedicine, delivering much-needed stroke expertise to hospitals and patients.

Objective: The aim of this paper was bring our expertise to confirm the importance of the application of a modern technology in medicine for improving diagnosis and care of the stroke.

Materials and Methods: Data was collected from 30 Italian participants during summer 2017, who selected in a big urban area, in one of the main stroke centers of the peninsula. From the teleconference, the neurologist can see the patient and perform the neurological examination and NIHSS again. The neurologists can answer the cross-consultation report, issuing a diagnosis if convenient. After this, the patient's diagnosis of stroke is confirmed and the trombolysis treatment can start.
\end{abstract}

Results: We demonstrated that using telestroke for six months, we had a timesaving of about 60-90 minutes to start thrombolysis. These results are in according to literature data.

Conclusions: Telestroke is a new approach to bringing expert stroke care to remote locations, with limited or no neurological expertise. The rising use of Telemedicine could actively improve diagnosis and care of stroke even if patients live in remote places.

Keywords: Technology; Telemedicine; Stroke

\section{Introduction}

Telemedicine includes a growing variety of applications and services, like video outputs, e-mail, cell phones or other forms of telecommunications technology [1]. From a historical point of view, the phone was the first communication means (1880) and it remains the most enduring for remote consultation. Besides the use of the phone, the first experience of telemedicine itself took place in the United States at the end of 1950 with the closed-circuit television that allowed the transmission of radiological images and pathological findings [2]. Starting more than forty years ago, with the experience of hospitals that have extended their assistance to patients in remote areas, the use of telemedicine has spread rapidly and is fully integrating into routine operations of hospitals, specialized departments, medical private and private care facilities [3]. 
The American Telemedicine Association has historically considered "Telemedicine" and "Telehealth" as interchangeable terms, indicating a broad definition of health care at distance, even if "Telehealth" is sometimes used to refer to a broader concept of health care at distance, which does not always involve clinical services [1]. Telemedicine founds a wide ground of application in neurological diseases and between these is very spread for Alzheimer disease [4].

The use of telestroke across the United States and Canada occurred in the late 1990s and early 2000s [5], but in Italy it comes later. It is one of the most frequently used and rapidly expanding applications of telemedicine, delivering much-needed stroke expertise to hospitals and patients [6]. At the moment the largest use of the telemedicine is the application of telemedicine for care of acute stroke, and neurology practitioners is probably the most important element of successful "telestroke" program [7].

Stroke is the leading cause of disability and death in developed countries. In Italy alone there are over 200,000 new cases of cerebrovascular disease annually - about $80 \%$ are ischemic strokes ( $25 \%$ of these recurrent strokes) and although hemorrhagic strokes occur less frequently (20\% of cases) they have a much higher mortality rate. Overall, mortality in the first 4 weeks following an ischemic stroke is $20 \%$, increasing to $30 \%$ in the first 12 months. Only $25 \%$ of patients who survive an ischemic stroke recover fully, while the majority become disabled and in a substantial proportion of these their disability is so severe that they are no longer selfsufficient and need to be managed in a chronic care setting [8].

Nowadays, clinical benefits of stroke units and thrombolysis in ischemic stroke are evidence-based. Critical factors to be solved are patient access difficulties to better therapies and advanced medical devices within the first 4.5 hours from the onset of symptoms. Therefore, time is important for the care of such patients: "Time is Brain".

\section{Objective}

Our working team has been part of a research project in which a telemedicine platform has been developed for the diagnosis and treatment of stroke episodes in static environments. The neurological team in the reference hospital has the technological means for replying to the consultations made from another hospital that lacks these kinds of specialists, in order to reduce time in stroke patient treatment procedures. Stoke Unit is a multidisciplinary team comprising of medical, nursing, physiotherapy and socialwork staff who coordinate their work through regular meetings $[9,10]$. All studies analyzed showed the importance of the stroke unit, because every minut is important, "every minut is brain" [11].

The study objective was bring our expertise to confirm the importance of the application of a modern technology in medicine for improving diagnosis and care of the stroke, to promote highimpact collaboration in the healthcare sector between emergencies and specialized medical teams during the acute phase stroke.

\section{Material and Methods}

Data was collected from 30 Italian participants during summer 2019, who selected in a big urban area, in one of the main stroke centers of the peninsula. The process starts when the patient or a family member calls the healthcare emergencies line and the patient may be transferred to the hospital. When patient data are confirmed, they must also confirm that the patient meets the criteria for inclusion (i.e. time of onset (when patient was last seen as normal) $<4.5$ hours before treatment would begin, age $>18$ years, National Institutes of Health Stroke Scale (NIHSS) scale between 5 and 25 (severe and moderately severe stroke), and that there is no exclusion absolute criteria, although individualizing circumstances "off-label" and, thus, classified as "Stroke Code". The physician alerts the neurologist at the reference hospital. They both can access a "videoconference virtual room" to perform telemedicine tasks. From the teleconference, the neurologist can see the patient and perform the neurological examination and NIHSS again. The neurologists can answer the cross-consultation report, issuing a diagnosis if convenient. After this, the patient's diagnosis of stroke is confirmed and the trombolysis treatment can start.

\section{Results}

The mean timesaving to start trombolysis was 75 minutes. Mean age was 73,2 years $\pm 11,7$ SD; 16 females and 14 males. We found a range of NIHSS from 7 to 23 (Table 1). In conclusion, we demonstrated that using telestroke in a few months, we had a timesaving of about 60-90 minutes to start trombolysis. These results are in according to literature data. Limitations of the trial are small sample and brief duration.

Table 1.

\begin{tabular}{|c|c|c|c|c|}
\hline Patient & Age & Sex & NIHSS & 7 \\
\hline n.1 & 75 & M & 9 & 65 \\
\hline n.2 & 61 & M & 16 & 70 \\
\hline n.3 & 81 & F & 12 & 93 \\
\hline n.4 & 83 & M & 14 & 72 \\
\hline n.6 & 63 & F & 10 & \\
\hline
\end{tabular}




\begin{tabular}{|c|c|c|c|c|}
\hline n.7 & 89 & $\mathrm{~F}$ & 7 & 74 \\
\hline n. 8 & 58 & M & 20 & 61 \\
\hline n.9 & 65 & $\mathrm{~F}$ & 15 & 85 \\
\hline n.10 & 63 & $\mathrm{M}$ & 21 & 89 \\
\hline n.11 & 59 & $\mathrm{M}$ & 13 & 65 \\
\hline n.12 & 84 & F & 10 & 77 \\
\hline n.13 & 73 & $\mathrm{~F}$ & 11 & 78 \\
\hline n.14 & 77 & $\mathrm{~F}$ & 24 & 82 \\
\hline n.15 & 80 & $\mathrm{~F}$ & 22 & 80 \\
\hline n.16 & 79 & $\mathrm{M}$ & 9 & 66 \\
\hline n.17 & 62 & M & 15 & 64 \\
\hline n.18 & 69 & $\mathrm{~F}$ & 20 & 84 \\
\hline n.19 & 87 & M & 11 & 71 \\
\hline n. 20 & 82 & $\mathrm{M}$ & 14 & 73 \\
\hline n. 21 & 78 & $\mathrm{M}$ & 18 & 60 \\
\hline n.22 & 45 & $\mathrm{~F}$ & 9 & 58 \\
\hline n.23 & 67 & M & 12 & 81 \\
\hline n.24 & 89 & $\mathrm{~F}$ & 8 & 75 \\
\hline n. 25 & 77 & $\mathrm{~F}$ & 23 & 79 \\
\hline n.26 & 49 & $\mathrm{~F}$ & 16 & 82 \\
\hline n.27 & 87 & $\mathrm{~F}$ & 21 & 79 \\
\hline n.28 & 81 & M & 13 & 73 \\
\hline n.29 & 82 & M & 20 & 79 \\
\hline n.30 & 66 & $\mathrm{~F}$ & 12 & 77 \\
\hline
\end{tabular}

\section{Conclusions}

Telemedicine's benefits range over different levels: (a) from individual patients, can support improvements in a patient's health and quality of life, particularly for those with chronic diseases, by enabling safer monitoring at home and reducing the number of hospital visits; (b) through healthcare systems as a whole, can help to address the shortage of healthcare professionals, particularly in sparsely populated areas (e.g. by providing remote consultations in ophthalmology), and can improve the efficiency, quality, and timeliness of healthcare service provision [12].

Prior studies have shown that the use of telemedicine for acute ischemic stroke is not only safe and effective, but it also increases the utilization of tPA, improving patient outcomes. These findings may encourage a systematization of telemedicine medical curricula to increase larger access to neurological consults. The application of new technologies to medicine had a recent exponential growth. Since the number of strokes among elderly people increases, the scientific community noticed that the rising use of telemedicine for stroke patients actively improve the care and the conditions of patients that live in remote places.

Telestroke is a new approach to bringing expert stroke care to remote locations, with limited or no neurological expertise.
Common obstacles to the practise of telestroke are medicolegal and economic issues. We concluded that further studies are recommended, primarily to improve the accuracy of the results.

\section{Declaration of Conflicting Interests}

The author(s) declared no potential conflicts of interest with respect to the research, authorship, and/or publication of this article.

\section{References}

1. American Telemedicine Association (ATA) (2008) What Is Telemedicine \& Telehealth?

2. Thrall JH, Boland G (1998) Telemedicine in practice. Semin Nucl Med 28(2):145-157.

3. Weiner JP (2012) Doctor-patient communication in the e-health era. Isr J Health Policy Res 1(1): 33.

4. Costanzo MC, Arcidiacono C, Rodolico A, Panebianco M, Aguglia E, et al. (2019) Diagnostic and Interventional implications of Telemedicine in Alzheimer's Disease and Mild Cognitive Impairment: a literature review. Int J Geriatr Psychiatry 35(1): 12-28.

5. Demaerschalk BM, Miley ML, Kiernan TE, Bobrow BJ, Corday DA (2009) Stroke telemedicine. Mayo Clin Proc 84(1): 53-64.

6. Camargo EC (2016) Stroke: highlights of selected articles. Stroke 47: 7.

7. Demaerschalk BM, Miley ML, Kiernan TE J, Bobrow BJ, Corday DA (2010) Stroke Telemedicine. Mayo Clin Proc 85(4): 400. 
8. Sacco S, Stracci F, Cerone D, Ricci S, Carolei A (2011) Epidemiology of stroke in Italy. Int J Stroke 6(3): 219-227.

9. European Stroke Organization (ESO) (2008) Guidelines for management of ischaemic stroke and transient ischaemic attack. Cerebrovasc Dis 25(5):457-507.

10. Stroke Unit Trialists' Collaboration (2007) Organised inpatient (stroke unit) care for stroke. Cochrane Database of Systematic Reviews.
11. Saver JL (2006) Time Is Brain- Quantified. Stroke 37: 263-266.

12. Torres Zenteno AH, Fernández F, Palomino García A, Moniche F, Escudero I, et al. (2016) Mobile platform for treatment of stroke: A case study of tele-assistance. Health Informatics J 22(3): 676-690. 